\title{
BMJ Open Efficacy and safety of left bundle branch area pacing versus right ventricular apex pacing in patients with atrioventricular block: study protocol for a randomised controlled trial
}

To cite: Wang Z, Du X, Cheng L, et al. Efficacy and safety of left bundle branch area pacing versus right ventricular apex pacing in patients with atrioventricular block: study protocol for a randomised controlled trial. BMJ Open 2021;11:e043603. doi:10.1136/ bmjopen-2020-043603

- Prepublication history for this paper is available online. To view these files, please visit the journal online (http://dx.doi org/10.1136/bmjopen-2020043603).

ZW and XD contributed equally.

Received 14 September 2020 Accepted 04 May 2021

Check for updates

(c) Author(s) (or their employer(s)) 2021. Re-use permitted under CC BY-NC. No commercial re-use. See rights and permissions. Published by BMJ.

${ }^{1}$ Department of Cardiology, Beijing An Zhen Hospital, Chaoyang-qu, China

${ }^{2}$ The First Hospital of Tsinghua University, Beijing, People's Republic of China

Correspondence to

Yongquan Wu;

wuyongquan67@163.com

\section{ABSTRACT}

Introduction Left bundle branch area pacing (LBBaP) is a relatively new approach for physiologic pacing. $A$ limited number of retrospective clinical studies, featuring small sample sizes, have shown that compared with right ventricular apex pacing (RVAP), the QRS duration of postoperative ECG in LBBaP patients is narrower and the cardiac systolic function is improved. However, there have been no randomised controlled trials (RCTs) evaluating the efficacy and safety of LBBaP in patients with atrioventricular block (AVB). Therefore, the current study intends to address the paucity in RCT data evaluating LBBaP versus RVAP in AVB patients.

Analysis and methods This study is a single-centre, randomised controlled superiority trial to be conducted at the Cardiac Centre of Beijing Anzhen Hospital. From January 2021 to December 2023, 210 consecutive AVB patients meeting the inclusion criteria and receiving pacemaker implantation will be enrolled. Participants will be randomly divided into an experimental group (LBBaP) and a control group (RVAP) at a 1:1 ratio. The primary outcome is left ventricular ejection fraction (LVEF), which will be assessed by echocardiography. The secondary outcomes in this study are synchrony of left ventricular systole, NT-proBNP, LVEDD, the 6-min walk distance, quality of life (SF-36 scale), all-cause mortality, cardiovascular death events, rehospitalisation rate and major complication rate.

Ethics and dissemination This study was approved by the Medical Ethics Committee of Beijing Anzhen Hospital (No. 2020021X). The results of the trial will be presented at national and international conferences. We hypothesise that compared with RVAP, LBBaP will be superior for treating patients with AVB. This trial will provide evidencebased suggestion for the majority of electrophysiologists in pacing implantation.

Trial registration ChiCTR2000034335.

\section{INTRODUCTION}

Hyman first introduced the concept of a pacemaker in 1932. ${ }^{1}$ Since the first pacemaker,
Strengths and limitations of this study

A risk assessment team will evaluate the entire study.

- Three non-investigators will use EpiData for data entry.

- Three experienced surgeons will perform the operations.

- A subgroup analysis will compare the efficacy and safety of left bundle branch area pacing and right ventricular apex pacing in two groups of patients with different ejection fractions.

- Since the echocardiographist can see where the ventricular lead is positioned, there is no way to blind the observation of ventricular function.

there has been no consensus on optimal location for placement of ventricular electrodes. Although previous studies have shown that traditional right ventricular apex pacing (RVAP) leads to interventricular and intraventricular dyssynchrony, this location also increases the risk of heart failure and atrial fibrillation. ${ }^{2}{ }^{3}$ Empirically, RVAP is still the most prolific approach for clinical pacemaker implantation. At present His bundle pacing (HBP) is considered to be the most physiological method. Compared with RVAP, HBP can maintain left and right ventricular electrical and mechanical relative synchronicities, and significantly reduce the incidence of heart failure and rehospitalisation rates in patients after pacemaker implantation. ${ }^{3-5}$ However, due to the special anatomical structure of the His bundle, adjacent HBP has higher threshold and should lead to events such as abnormal sensing and atrial cross perception. Meanwhile, there is limited therapeutic 
effect in patients receiving blocks below the His bundle, ${ }^{6}$ leading to a IIa guideline recommendation.

While left bundle branch area pacing (LBBaP) has been developed on the basis of HBP, LBBaP is able to overcome some of the clinical limitations of traditional HBP. $^{7-10}$ In 2017, Huang et $a l^{7}$ proposed the concept of $\mathrm{LBBaP}$, and the results of LBBaP treatment in 210 patients with pacing indications were presented at the 2019 Heart Rhythm Society conference. The threshold was stable at 1-year of follow-up, and minor complications occurred in only five patients. No surgical complications such as lead dislocation, lead infection or stroke were reported at long term follow-up. ${ }^{9}$ These data are suggestive that LBBaP is safe and feasible. While a retrospective trial comparing LBBaP and RVAP at 3 months follow-up showed stable pacing thresholds and no adverse events in both groups, the LBBaP group was associated with a narrower QRS duration compared with the RVAP group. ${ }^{10}$

Our Centre has previously published data on LBBaP implantation using a New Nine Partition Method. The results at 1-year follow-up showed that the duration of QRS was narrow, the pacing threshold was low, the parameters (voltage, sensing and impedance)were stable, and there were no surgical complications such as lead dislocation, lead infection or stroke. ${ }^{11}$

Furthermore, our Centre has completed a study that contrasts LBBaP and RVAP in patients with atrioventricular block (AVB) and normal cardiac function. ${ }^{12}$ Recent follow-up has shown that LBBaP can improve left ventricular synchronisation and cardiac function. ${ }^{12}$ Although existing studies have shown that LBBaP can improve cardiac function in AVB patients, avoid the risk of ventricular desynchronisation caused by RVAP and overcome some limitations associated with HBP, these prior data sets comprise small sample sizes and observational designs. The field still lacks high-quality evidence, limiting the recommendations in clinical guidelines and clinical utility of LBBaP.

On this basis, our Centre intends to conduct a randomised controlled trial in AVB patients to compare the effects of LBBaP and traditional RVAP on electromechanical synchronisation and long-term prognosis. This study will be designed to explore the efficacy and safety of LBBaP treatment in patients with AVB. This study is expected to provide strong evidence-based data to inform clinical guidelines for pacing in AVB patients.

\section{RESEARCH QUESTIONS AND STUDY HYPOTHESIS Objective}

This study will address whether LBBaP is superior to RVAP with regard to the following characteristics: (1) LVEF, (2) NT-proBNP, (3) synchrony of left ventricular systole, (4) left ventricular end diastolic diameter (LVEDD), (5) the 6-min walk distance, (6) quality of life (SF-36 scale), (7) all-cause mortality, (8) cardiovascular death events, (9) the rehospitalisation rate, (10) lead dislocation and infection, (11) other rehospitalisation rate, (12) time of ventricular lead implantation, (13) time of operation, (14) fluoroscopy time of ventricular lead implantation, (15) time of fluoroscopy, (16) pacing parameters, (17) thickness of interventricular septum, (18) electrode entry depth and (19) distance between electrode and base of tricuspid valve.

\section{Hypothesis}

We hypothesise that LVEF in LBBaP is superior compared with RVAP in patients with AVB.

\section{METHODS AND ANALYSIS \\ Study design}

A single-centre, randomised controlled superiority trial will be enforced at the Cardiac Centre of Beijing Anzhen Hospital. From January 2021 to December 2023, consecutive patients with AVB who undergo permanent pacemaker implantation and meet the eligibility criteria will be enrolled in this study (figure 1). This study has been approved by the Beijing Anzhen Hospital Medical Ethics Committee. Participants evaluated in this trial will sign an informed consent form in which they will agree to participate in the trial while they are in the hospital as well as during follow-up.

\section{Subjects}

\section{Inclusion criteria}

1. Adults aged $18-80$ years.

2. Patients with AVB who meet indications for pacemaker implantation.

3. Estimated percentage of ventricular pacing $\geq 50 \%$.

4. Ability to provide informed consent.

\section{Exclusion criteria}

1. Failure to cooperate with follow-up.

2. Persistent atrial fibrillation.

3. Patients with heart failure (LVEF) $\leq 35 \%$.

4. Complete heart block.

5. Estimated glomerular filtration rate $<30 \mathrm{~mL} / \mathrm{min}$.

6. Septic shock.

7. Advanced malignant tumour.

8. Pregnancy or prepared to get pregnant.

9. Cardiac tamponade or major haemopericardium.

\section{Study intervention}

\section{RVAP group}

In patients receiving RVAP, the conventional apical position of a passive-fixation ventricular lead tip will be fluoroscopically superimposed with the diaphragm. The subclavicular vein or axillary vein will be punctured, and the right ventricular lead will be delivered to the right ventricular apex. The lead will be placed within the appropriate position of the cardiac chamber. Perception, threshold and impedance will be evaluated, and the following parameters are required for the implantation to be considered successful: pacing threshold of $<1.0 \mathrm{~V}$, an R-wave of $>5.0 \mathrm{mV}$ and an impedance of $500-1500$ 


\section{From $1 / 2021$ to $12 / 2023$, the patients with AVB who need instrumentation will be enrolled in this trial}

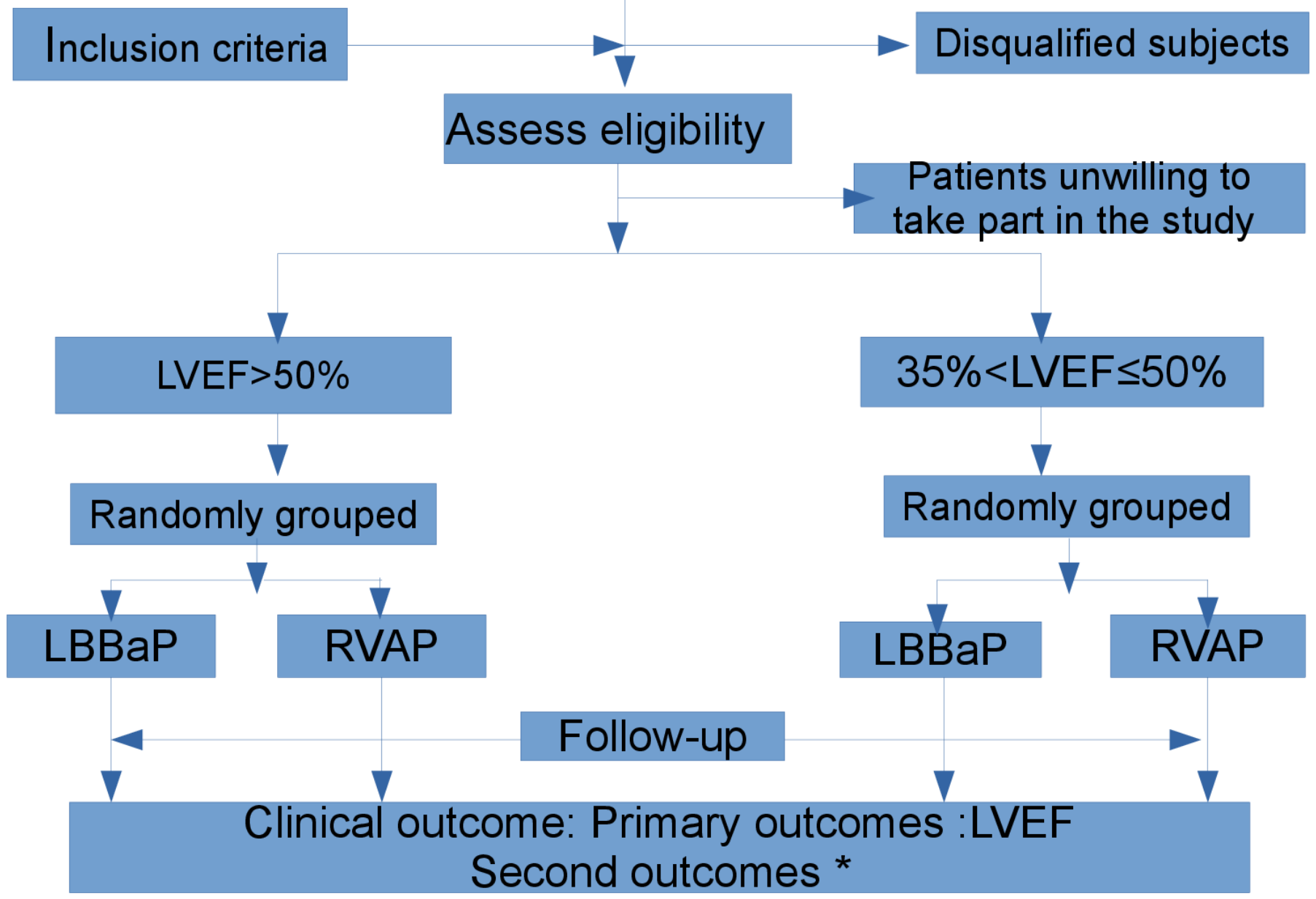

Figure 1 Flow diagram of enrolment, intervention and assessments. *Efficacy, safety and other outcomes. AVB, atrioventricular block; LBBaP, left bundle branch area pacing; LVEF, left ventricular ejection fraction; RVAP, right ventricular apex pacing.

V. If RVAP cannot be achieved, patients will go through LBBaP implantation or surgery.

\section{LBBaP group}

\section{Implantation method}

The LBBaP lead will be implanted using the New Nine Partition Method. ${ }^{11}$ During the procedure, a bedside patient monitor will record lead V1 signal. Lead V of the monitor is affixed to the V1 position for 12-lead ECGs. Guided by the C315 sheath (Medtronic, Minneapolis, Minnesota), the ventricular pacing lead (model 3830, Medtronic) will be inserted into the left bundle branch area. During the procedure, Medtronic pacemaker programmer will test the threshold. If LBBaP cannot be achieved, patients will undergo RVAP implantation or surgery.

\section{Definition of a successful LBBaP}

1. Under fluoroscopy, the 3830 lead was inserted into the left bundle branch area.
2. Unipolar pacing will demonstrate narrowed QRS complex $(<130 \mathrm{~ms}$ in the present study).

3. S-pLVAT (in leads V4-V6, the duration from the stimulus to the peak of the $\mathrm{R}$ wave) will be $<90 \mathrm{~ms} .{ }^{12}$

\section{Randomisation and masking}

A random sequence is generated by a central computer system, and subjects meeting the registration criteria will be assigned to the LBBaP or RVAP groups in a 1:1 ratio. Patients' medical information will be kept confidential.

\section{Study procedures}

Screening

AVB patients receiving treatment at the Cardiac Centre of Beijing Anzhen Hospital from January 2021 to December 2023 will be screened using the study inclusion and exclusion criteria. Patients will be divided into two subgroups according to LVEF: $\mathrm{LVEF}>50 \%$ group and LVEF reduction group $(35 \%<\mathrm{LVEF} \leq 50 \%)$. Once patients are enrolled, baseline characteristics will be gathered using case report 


\begin{tabular}{llll}
\hline Table 1 Details of follow-up & & & \\
\hline Follow-up & Inpatient & Outpatient clinic & Telephone follow-up \\
\hline Visiting time & Preoperation & $\begin{array}{c}\mathbf{1 , 1 2 , 2 4} \text { months } \\
\text { postoperation }\end{array}$ & $\begin{array}{c}\mathbf{3}, \mathbf{6}, \mathbf{1 8} \text { months } \\
\text { postoperation }\end{array}$ \\
\hline Basic medical history & & & \\
Sign informed consent & $\sqrt{ }$ & \\
Medical history & $\sqrt{ }$ & & \\
Vital sign & $\sqrt{ }$ & $\sqrt{ }$ & \\
Comorbidity and mortality & & \\
Assistant examination & & $\sqrt{ }$ \\
Routine blood test & $\sqrt{ }$ & \\
ECG & $\sqrt{ }$ & $\sqrt{ }$ \\
Holter & $\sqrt{ }$ & $\sqrt{ }$ \\
Echo & $\sqrt{ }$ & $\sqrt{ }$ \\
6-minute walking test & $\sqrt{ }$ & $\sqrt{ }$ \\
Quality of life (SF-36 scale) & $\sqrt{ }$ & $\sqrt{ }$ \\
Programme under echocardiography & & $\sqrt{ }$ \\
\hline
\end{tabular}

forms (CRF). Age, sex, body mass index (BMI), history (eg, cardiomyopathy, coronary heart disease, hypertension, diabetes mellitus), medication history, 6-min walking test, blood sample test and Holter monitoring results will be included. Echocardiography and a surface ECG will be evaluated separately by two experienced doctors. Interventricular thickness will be documented.

\section{Data collection}

During the procedure, specialised personnel will collect intraoperative data. Information on complications, X-ray duration, radiation dosage as well as detailed information on the pacemaker and pacing lead, pacemaker parameters (eg, voltage, sensing, impedance), and QRS duration will be recorded.

\section{Randomization and follow-up visits}

Randomisation will be completed between 1 January 2021 and 31 December 2022, with a 24-month follow-up duration. Outpatient follow-up will be evaluated at 1, 12 and 24 months postindex procedure. Routine blood tests, echocardiography and surface electrograms will be performed and telephone follow-up will be performed at 3,6 and 18 months postindex procedure. The rehospitalisation rate will be described (table 1).

\section{Risk management}

The risk management team will assess the entire study. All mortality and morbidity events will be monitored. If patients occur any cardiovascular disease, they will be admitted to hospital. We will stop the experiment and give them appropriate treatment.

\section{Patient and public involvement}

Patients or the public were not involved in the design, conduct, reporting or dissemination of the research.

\section{Outcomes}

Primary outcomes

LVEF is critical for evaluating heart function. LVEF will be tested using echocardiography at 1, 12 and 24 months postoperation by experienced doctors.

\section{Secondary outcomes}

Efficacy outcomes

NT-proBNP, synchrony of left ventricular systole, LVEDD, 6 -min walk test and quality of life (SF-36 scale). All-cause mortality, cardiovascular death, rehospitalisation rate of heart failure and other rehospitalisation rate.

\section{Safety outcomes}

Lead dislocation, lead infection. Major complication rates: cardiac perforation, haemopericardium, malignant arrhythmias, sudden cardiac death, and acute pulmonary oedema.

\section{Other outcomes}

Time of ventricular lead implantation, time of operation, fluoroscopy time of ventricular lead implantation, time of fluoroscopy, pacing parameters, thickness of interventricular septum, electrode entry depth, distance between electrode and base of the tricuspid valve.

\section{Data management and monitoring}

\section{Before the trial}

The trialists will be trained prior to the study. The randomisation will be conducted by a specialised team, the surgical team will adhere to technical standards and the echocardiography team will be responsible for echocardiographic assessment. The data processing team will use EpiData, as well as CRF tables to standardise the input. There will be no cross-communication among the specialised teams, including the operation and data entry teams. 
All personnel will sign a patient privacy confidentiality agreement.

\section{During the trial}

As described above, patients will be randomly divided into two groups. The rate of patient crossover will be controlled to less than 5\%. Three surgeons will be randomised in performing the surgical treatment (only operators who had performed at least 105 RVAP and 105 LBBaP will perform the procedure). Three reviewers will evaluate relevant indicators, two specialists will collect and sort data, and one specialist will examine the data. There is no intersection between the data collectors and the operators.

\section{Interim analyses}

An interim analysis will be conducted in December 2021 to evaluate the surgical success rate, complication rate, missing data rate and crossover rate. If the complication rate is higher than $10 \%$, the trial will be stopped early.

\section{After the trial}

Researchers will collect and retain the informed consent forms, CRFs and other research documents. Clinical outcome data are to be collected manually from electronic medical records.

\section{Sample size calculation}

This study is designed to evaluate the efficacy and safety of LBBaP in the treatment of patients with AVB. The main study outcome is left ventricular ejection fraction (LVEF). According to prior reports, the 1-year improvement of LVEF in patients treated with LBBaP for AVB was higher compared with traditional RVAP (0.06). The SD of LVEF in the LBBaP group is estimated at 0.17 , and the $\mathrm{SD}$ of LVEF in the RVAP group is 0.13 . The superiority margin is $1 / 5(0.012)$ of the difference between the two groups, indicating that LBBaP is superior to RVAP. The sample size difference between the two groups was estimated by PASS V.15.0 (superiority test). The significance level $(\alpha)$ was set 0.025 , and the assurance level $(1-\beta)$ was 0.90 . At least 94 cases need to be enrolled in each of the two groups, for a total of 188 cases. Loss to follow-up is considered less than $10 \%$. Therefore, 207 cases will be enrolled in the proposed study.

\section{LVEF $>\mathbf{5 0} \%$ group}

According to previous reports, the improvement of LVEF in the LBBaP group was higher compared with the RVAP group at 1 year $(0.04)$. The SD of LVEF in the LBBaP group is estimated at 0.08 and the SD of LVEF in the RVAP group is 0.080 . The superiority margin is set at $1 / 5$ $(0.008)$ of the difference between the two groups, indicating that LBBaP is superior to RVAP. At least 60 cases need to be enrolled in each group, for a total of 120 cases. Loss to follow-up is considered less than $10 \%$. Therefore, 132 cases will be enrolled for the proposed subgroup analysis.

\section{LVEF reduction group ( $35 \%<\mathrm{LVEF} \leq 50 \%)$}

According to previous reports, the improvement of LVEF in the LBBaP group was higher than in the RVAP group at 1 year (0.09). The SD of LVEF in the LBBaP group is estimated at 0.110 and the SD of LVEF in the RVAP group is 0.130 . The superiority margin was set at $1 / 5$ $(0.018)$ of the difference between the two groups, indicating that LBBaP is superior to RVAP. At least 28 cases need to be enrolled in each group, for a total of 56 cases. Loss to follow-up is considered less than $10 \%$. Therefore, 62 cases will be enrolled for the proposed subgroup analysis.

The total sample size for the primary analysis is estimated at 207 cases, and the subgroup analysis requires 194 cases. The maximum sample size (ie, 210 patients) was selected for inclusion in the study.

\section{Statistical analysis}

Three non-investigators will use EpiData for data entry. Two independent statisticians will complete the statistical analysis. The LBBaP group includes: (1) complete LBBaP treatment or (2) failure to be treated with LBBaP or eventually treated by RVAP. Definition of LBBaP treatment failure: (a) failure to meet LBBaP parameter criteria; (b) ventricular electrodes cannot be implanted; (c) pacing threshold after implantation greater than 3V/0.4 ms. The RVAP group includes as follows: (3) complete RVAP treatment or (4) failure to be treated with RVAP or eventually treated by LBBaP. Definition of RVAP treatment failure: (a) ventricular electrodes cannot be implanted; or (b) the right ventricular electrode pacing threshold is greater than $3 \mathrm{~V}$ at $0.4 \mathrm{~ms}$. The intention-to-treat set is used to compare $(1)+(2)$ with $(3)+(4)$. The per-protocol analysis set is used to compare (1) with (3). The data will be analysed by SPSS statistics (IBM, V.23). Normally distributed continuous variables (age, sex, BMI, blood sample test results, NT-proBNP, duration of the QRS complex, LVEF, LVEDD, 6-min walk test and SF-36 scale) will be expressed as the mean \pm SD and nonuniformly distributed data will be expressed as the median (Q1 and Q3). For the comparison of the mean between groups (history and medication history), independent sample t-test will be used for normal distribution data, and Mann-Whitney $\mathrm{U}$ test will be used for non-uniform distribution data. A Kaplan Meier curve will be used to determine the events rate between the two groups over time, and the log rank test will be used to compare two groups. The Cox proportional risk model will be used to calculate the HR. A p-value $<0.05$ will be considered statistically significant.

\section{Planned subgroup analyses}

1. Sex.

2. Ejection fraction.

3. Duration of QRS complex,

4. Six-minute walking test.

5. Quality of life (SF-36 scale). 


\section{Bias}

Patients will be consecutively enrolled from the Cardiac Centre of Beijing Anzhen Hospital. In total, 210 patients with AVB undergoing LBBaP or RVAP will be included. A randomisation sequence will be generated by a centralised computer system, and participants will be assigned to the LBBAP or RVAP group at a 1:1 ratio.

\section{Ethics and dissemination}

This study has been approved by the Beijing Anzhen Hospital Medical Ethics Committee (No. 2020021x) and abides by the principles of the Helsinki Declaration. Participants evaluated in this trial will provide informed consent and sign an involvement statement in which they will agree to participate in the trial while they are in the hospital as well as during follow-up. All research documents will be collected and stored at Beijing Anzhen Hospital.

This single-centre, randomised controlled superiority trial will be conducted at the Cardiac Centre of Beijing Anzhen Hospital from January 2021 to December 2023. After this trial, the results of this study will be shown at domestic and international conferences for research and further study.

Correction notice Since this article was first published online, a funding statement has been added.

Contributors ZW and XD are joint first authors. YW and JZ obtained funding. ZW and $X D$ designed the study. JunC, XD and ZW collected the data. CL and JC were involved in data cleaning, mortality follow-up and verification. XW and $\mathrm{FH}$ analysed the data. RD drafted the manuscript. ZL contributed to the interpretation of the results and critical revision of the manuscript for important intellectual content and approved the final version of the manuscript. All authors have read and approved the final manuscript. YW and $\mathrm{JZ}$ are the study guarantors.

Funding This study was supported by the Beijing Hospitals Authority Clinical Medicine Development of Special Funding Support (grant number XMLX202112).

Competing interests None declared.

Patient and public involvement Patients and/or the public were not involved in the design, or conduct, or reporting or dissemination plans of this research.

Patient consent for publication Not required.

Provenance and peer review Not commissioned; externally peer reviewed.
Open access This is an open access article distributed in accordance with the Creative Commons Attribution Non Commercial (CC BY-NC 4.0) license, which permits others to distribute, remix, adapt, build upon this work non-commercially, and license their derivative works on different terms, provided the original work is properly cited, appropriate credit is given, any changes made indicated, and the use is non-commercial. See: http://creativecommons.org/licenses/by-nc/4.0/.

ORCID iDs

Xiao Du http://orcid.org/0000-0002-3133-3532

Yongquan Wu http://orcid.org/0000-0001-9156-4669

\section{REFERENCES}

1 Lown B, Kosowsky BD. Artificial cardiac pacemakers. II. N Engl J Med 1970;283:971-7.

2 Abdelrahman M, Subzposh FA, Beer D, et al. Clinical outcomes of His bundle pacing compared to right ventricular pacing. J Am Coll Cardiol 2018:71:2319-30.

3 Ye Y, Zhang Z, Sheng X, et al. Upgrade to his bundle pacing in pacing-dependent patients referred for pulse generator change: feasibility and intermediate term follow up. Int $\mathrm{J}$ Cardiol 2018;260:88-92.

4 Lustgarten DL, Crespo EM, Arkhipova-Jenkins I, et al. His-bundle pacing versus biventricular pacing in cardiac resynchronization therapy patients: a crossover design comparison. Heart Rhythm 2015;12:1548-57.

5 Sharma PS, Dandamudi G, Herweg B, et al. Permanent Hisbundle pacing as an alternative to biventricular pacing for cardiac resynchronization therapy: a multicenter experience. Heart Rhythm 2018;15:413-20.

6 Zanon F, Ellenbogen KA, Dandamudi G, et al. Permanent His-bundle pacing: a systematic literature review and meta-analysis. Europace 2018;20:1819-26.

7 Huang W, Su L, Wu S, et al. A novel pacing strategy with low and stable output: pacing the left bundle branch immediately beyond the conduction block. Can J Cardiol 2017;33:1736.e1-1736.e3.

8 Cheng L-T, Zhang J-M, Wang Z-F, et al. Recent approaches to HisPurkinje system pacing. Chin Med J 2019;132:190-6.

9 Vijayaraman P, Subzposh FA, Naperkowski A, et al. Prospective evaluation of feasibility and electrophysiologic and echocardiographic characteristics of left bundle branch area pacing. Heart Rhythm 2019;16:1774-82.

10 Chen K, Li Y, Dai Y, et al. Comparison of electrocardiogram characteristics and pacing parameters between left bundle branch pacing and right ventricular pacing in patients receiving pacemaker therapy. Europace 2019;21:673-80.

11 Zhang J, Wang Z, Zu L, et al. Simplifying physiological left bundle branch area pacing using a new Nine-Partition method. Can $J$ Cardiol 2021;37:329-338.

12 Zhang J, Wang Z, Cheng L, et al. Immediate clinical outcomes of left bundle branch area pacing vs conventional right ventricular pacing. Clin Cardiol 2019;42:768-73. 
Correction: Efficacy and safety of left bundle branch area pacing versus right ventricular apex pacing in patients with atrioventricular block: study protocol for a randomised controlled trial

Wang Z, Du X, Cheng L, et al. Efficacy and safety of left bundle branch area pacing versus right ventricular apex pacing in patients with atrioventricular block: study protocol for a randomised controlled trial. BMJ Open 2021;11:e43603. doi:10.1136/ bmjopen-2020-043603

Since this article was first published online, the following funding information has been added.This study was supported by the Beijing Hospitals Authority Clinical Medicine Development of Special Funding Support (grant number XMLX202112).

Open access This is an open access article distributed in accordance with the Creative Commons Attribution Non Commercial (CC BY-NC 4.0) license, which permits others to distribute, remix, adapt, build upon this work non-commercially, and license their derivative works on different terms, provided the original work is properly cited, appropriate credit is given, any changes made indicated, and the use is non-commercial. See: http://creativecommons.org/licenses/by-nc/4.0/.

(c) Author(s) (or their employer(s)) 2021. Re-use permitted under CC BY-NC. No commercial re-use. See rights and permissions. Published by BMJ.

BMJ Open 2021;11:e043603corr1. doi:10.1136/bmjopen-2020-043603corr1 